\title{
Aualiação e feedback de desempenho de estudantes na educação a distância
}

\section{Evaluación y feedback del desempeño de estudiantes en la educación a distancia Assessment and Feedback of Students' Performance in Distance Education Courses}

\author{
Aline B. Archer*, Ana C. Crispim*, Roberto M. Cruz* \\ *Universidade Federal de Santa Catarina, Santa Catarina, Brasil.
}

Doi: http://dx.doi.org/10.12804/ap134.3.2016.03

\section{Resumo}

Avaliação de desempenho é uma estratégia utilizada para investigar o desempenho de estudantes no contexto educacional presencial ou a distância. Este artigo tem por finalidade demonstrar os aspectos que constituem o processo de avaliação de desempenho de estudante e, especialmente, o uso de feedback na educação a distância. Foi realizada uma revisão teórica sustentada em duas perspectivas de análise: conceitos de avaliação de desempenho e o processo de feedback do desempenho do estudante como parte constituinte da avaliação de desempenho na educação a distância. Conceitos sobre avaliação de desempenho são diversos na literatura científica. Alguns enfatizam a interpretação da expressão numérica do desempenho do estudante, outras recaem seu foco no uso de feedback como meio de aperfeiçoamento do desempenho. A avaliação de desempenho na educação a distância decorre de processos de interação entre o tutor e o estudante, mediados por tecnologias, e dependem de feedback para promover a participação nas atividades de ensino-aprendizagem. Nessa modalidade, o uso de feedback do desempenho dos estudantes é considerado aspecto relevante à orientação do processo educativo e ao aperfeiçoamento do desempenho social e profissional. A estratégia de uso de feedback imediato e informativo, com recursos que facilitem ao estudante acompanhar o desenvolvimento de seu desempenho, auxilia-o na identificação do que necessita ser modificado e aperfeiçoado ao longo do processo educativo. Palavras-chave: avaliação de desempenho; feedback; educação a distância.

\section{fbstract}

Performance assessment is a strategy to investigate students' performance in the educational field.This paper aims to demonstrate what composes the performance assessment process, and especially, the feedback application in distance education. To understand these

* Aline B. Archer, Ana C. Crispim, Roberto M. Cruz, Departamento de Psicologia, Universidade Federal de Santa Catarina, Santa Catarina, Brasil.

Essa pesquisa foi apoiada em partes pela Coordenação de Aperfeiçoamento de Pessoal de Nível Superior (CAPES) com bolsas para as duas autoras do artigo.

A correspondência relacionada com este artigo deve ser direcionada a Aline B. Archer, Laboratório Fator Humano, Departamento de Psicologia, Universidade Federal de Santa Catarina, Campus Universitário Reitor João David Ferreira Lima, Florianópolis, Santa Catarina, Brasil, 88040-900. Correio eletrônico: alinearcherr@gmail.com

Cómo citar este artículo: Archer, A. B., Crispim, A. C., Cruz, R. M. (2016). Avaliação e feedback de desempenho de estudantes na educação a distância. Avances en Psicología Latinoamericana, 34(3), 473-485. doi: http://dx.doi.org/10.12804/apl34.3.2016.03 
phenomena, it was performed a theoretical review that was supported by two perspectives: performance assessment concepts and the process of performance feedback to the student as a component of the performance assessment in distance education. There is a variety of concepts about performance assessment in the scientific literature. Some definitions emphasize the numeric expression interpretation of the student's performance, and others focus on feedback's application as a resource to improve the student's performance. The performance assessment in distance education happens because of the interaction processes between teacher and student, which are mediated by technologies, and depend on feedback to promote teaching and learning activities. In distance education modality, the feedback to the students' performance is a relevant issue for the educational orientation process, and for the social and professional performance improvement. The immediate and informative feedback strategy, which uses facilitating resources for the student follow his performance development, assists on the identification of what needs to be modified and refined through the educational process.

Keywords: performance assessment; feedback; distance education.

\section{Resumen}

La evaluación de desempeño es una estrategia utilizada para investigar el desempeño de estudiantes en el contexto educacional presencial o a distancia. Este artículo tiene por finalidad demostrar los aspectos que constituyen el proceso de evaluación de desempeño del estudiante y, especialmente, el uso de feedback en la educación a distancia. Se realizó una revisión teórica sustentada en dos perspectivas de análisis: conceptos de evaluación de desempeño y el proceso de feedback del desempeño del estudiante como parte constituyente de la evaluación de desempeño en la educación a distancia. Los conceptos sobre evaluación de desempeño son diversos en la literatura científica. Algunos enfatizan la interpretación de la expresión numérica del desempeño del estudiante, otras se enfocan en el uso de feedback como medio de perfeccionamiento del desempeño. La evaluación de desempeño en la educación a distancia transcurre entre procesos de interacción entre el tutor y el estudiante, mediados por tecnologías y dependen de feedback para promover la participación en las actividades de enseñanza-aprendizaje. En esa modalidad, el uso de feedback del desempeño de los estudiantes es considerado un aspecto relevante en la orientación del proceso educativo y en el perfeccionamiento del desempeño social y profesional. La estrategia de uso de feedback inmediato e informativo tiene recursos que facilitan al estudiante acompañar el desarrollo de su desempeño, y ayudarlo en la identificación de lo que necesita ser modificado y perfeccionado a lo largo del proceso educativo.

Palabras clave: evaluación de desempeño; feedback; educación a distancia.

Maria e João são colegas universitários e ela solicitou que ele avaliasse seu artigo: Maria: como você avalia o meu artigo? João: Está muito bom! Maria: Mas eu pedi para você avaliá-lo e não dar apenas sua opinião sobre ele.

No diálogo entre Maria e João é possível perceber que ambos os personagens estão nomeando da mesma forma processos distintos. João compreende avaliação sob um ponto de vista: ao emitir um juízo de valor, entende que avaliou o artigo. Mas, para Maria, o que João fez não consistiu em avaliação, tanto que indicou ao João o não cumprimento do que foi solicitado. Mas o que Maria entende por avaliação? Concepções distintas e nomeadas com a mesma expressão, quando não explicitadas, dificultam a comunicação entre as pessoas. Maus entendidos entre as pessoas em ambiente profissional, muitas vezes, têm implicações danosas para elas e eventualmente para outras. Divergências sobre o conceito de avaliar e a importância da avaliação no ambiente educacional para gestores, professores e estudantes, indicam a necessidade de aperfeiçoar a compreensão sobre o papel da avaliação em 
processos de ensino-aprendizagem, para superar os "velhos" estereótipos que tentam associar perspectivas de avaliar como "ganhar um rótulo".

O processo de avaliar tem como produto principal a produção de informações, oriunda da comparação entre o que ocorre na realidade e alguma situação tomada como referencial, o que possibilita tomada de decisões acerca do objeto avaliado ou de outros aspectos relacionados a ele (De la Orden, 2000). No contexto de educação, o feedback é caracterizado como um dos procedimentos utilizados decorrentes da decisão sobre o que foi avaliado. O conceito de feedback pode ser compreendido como a comunicação de informações ao estudante pelo professor com a finalidade de melhorar seu desempenho (Shute, 2007), o qual consiste em comportamentos que podem ser observados diretamente ou indiretamente (Mager, 1981) e no grau de seu desenvolvimento em relação a um comportamento esperado (Lamonato, 2011).

Fornecer feedback informativo e contínuo aos estudantes acerca de aspectos relevantes do seu desempenho é uma característica constituinte do processo de avaliação de desempenho no contexto da educação em geral e, especialmente, na educação a distância (EaD). O uso de feedback do desempenho dos estudantes é relevante, não somente para a orientação do estudante quanto ao seu desenvolvimento, mas também como uma estratégia importante de gestão do processo de ensino-aprendizagem.

O uso inapropriado do feedback, ou a não utilização de feedback em avaliações de desempenho implica no aumento da probabilidade de que o egresso de um programa de ensino apresente repertório comportamental limitado que pode vir a trazer consequências para qualidade de sua atuação profissional. É de responsabilidade social e econômica dos programas de educação a distância garantir que o resultado do processo de avaliação corresponda à qualidade do objeto avaliado, uma vez que na avaliação de desempenho é necessário identificar o desempenho do estudante com precisão, a fim de possibilitar o seu aperfeiçoamento, durante o processo de capacitação e ao longo de sua vida profissional. Quando o estudante recebe uma certificação, a qual refere o reconhecimento formal de comportamentos desenvolvidos, é atestado também que esse estudante está apto para intervir socialmente com base no que aprendeu em curso destinado à formação ou capacitação (Chacón, 1994; Alexim \& Lopes, 2003).

Como decorrência social da educação, é necessário salientar que as pessoas necessitam aprender e aperfeiçoar seus comportamentos para promover benefícios à sociedade em qual atuam. Atuar profissionalmente implica mais do que exercer práticas existentes e atender demandas de organizações ou de pessoas isoladamente, é necessário intervir nos problemas e necessidades sociais para modificar situações de forma a promover o máximo de benefícios possíveis para as pessoas envolvidas nelas (Botomé \& Kubo, 2002). Uma das funções da educação é possibilitar que mais cidadãos tenham acesso ao conhecimento e que, com base nele, busque novas e melhores soluções para os problemas sociais. Segundo Botomé (1981) e Ribeiro (1969), por meio da educação, as pessoas necessitam aprender a agir de modo que os resultados de suas ações sejam significativos para aqueles que compõem a sociedade da qual cada um faz parte.

Dada a relevância social e científica de se realizar avaliações de desempenho do estudante e do uso apropriado do feedback nesse processo, este artigo possui como finalidade demonstrar os aspectos que constituem o processo de avaliação de desempenho de estudante e, especialmente, o uso de feedback na educação a distância. Para compreender esses fenômenos, foi realizada uma revisão teórica, que está apresentada em duas partes: "Avaliar e medir: perspectivas conceituais na avaliação de desempenho" e "Feedback do desempenho do estudante como parte constituinte da avaliação de desempenho na educação a distância". 


\section{Avaliar e medir: perspectivas conceituais na avaliação de desempenho}

Na literatura científica, é comum encontrar uma diversidade de definições acerca de avaliação e de medida. Algumas são sobrepostas, outras complementares ou divergentes entre si. Na literatura da psicologia, especialmente no campo designado como Psicometria, autores como Cruz, Alchieri e Sardá (2002), Pasquali (2011) e Caixeta e Silva (2014) ressaltam que os processos de medir e avaliar são complementares e indissociáveis, sustentados pelos processos metodológicos de observar, descrever e explicar fenômenos psicológicos, suas peculiaridades e relação com o contexto. Para isso, é utilizada a teoria da medida, a qual entende a mensuração como o processo de designar números a atributos quantificáveis de acordo com uma "régua” (McDonald, 2014) e preza pela preservação do isomorfismo entre as propriedades numéricas e os atributos do objeto na empiria (Pasquali, 2011). Já avaliar é investigar as características dos indivíduos por meio de testes, entrevistas e outros procedimentos que sejam pertinentes (McDonald, 2014). Assim, na psicologia, avaliar aspectos do comportamento humano é estimar as suas qualidades e variações, com base em suposições teóricas sobre a sua dimensionalidade na vida das pessoas.

Na educação, a discussão acerca da avaliação e da medida é voltada principalmente para o desempenho do estudante. Nesse âmbito, há autores que diferenciam "medida" da "avaliação", tendo como critério de distinção a atribuição de valores numéricos ao objeto de interesse para a medida e a atribuição de valores qualitativos ao objeto de interesse, no caso da avaliação. Esteves (1973), Haydt (1997) e Santa'anna (2002) entendem o processo de medir como a determinação da extensão, dimensões, quantidade, capacidade ou grau de alguma característica do objeto. A medida é expressa em número, tendo como base um sistema de unidades convencionais. Para os mesmos autores, a avaliação consiste em interpretar os resultados da medida, por meio de julgamentos, descrições e opiniões, com diferentes graus de precisão que esta interpretação pode ser feita. Ocorre predominantemente por meio de análises qualitativas. Por outro lado, Luckesi (2011) e Botomé e Rizzon (1997), embora orientados por matrizes epistemológicas distintas, discutem o processo de avaliar desempenho, no contexto educacional e da psicologia, como um processo que implica em mais do que atribuir um valor quantitativo ou qualitativo a um objeto. Para os autores, a avaliação é constituída pelo processo de intervenção do professor em direção ao desenvolvimento do desempenho do estudante.

Luckesi (2011) afirma que a avaliação de desempenho não possui finalidade em si. O processo de avaliar consiste na atribuição de qualidades, com base em dados relevantes, do desempenho do estudante para que sejam tomadas decisões em direção ao seu desenvolvimento. Esse processo envolve duas etapas articuladas e indissociáveis: a) diagnosticar; b) intervir com objetivo de melhorar os resultados, caso necessário. Diagnosticar é um processo que envolve a qualificação da realidade por meio de sua descrição com base em dados relevantes. A qualificação é obtida pela comparação entre realidade descrita e um critério assumido como qualidade desejada, seja ela expressa em números ou palavras. O processo de qualificar em si consiste em uma tomada de posição acerca do objeto da avaliação que, por sua vez, permite uma tomada de decisão em direção ao desenvolvimento do desempenho do estudante. O objetivo da intervenção é corrigir as ações em curso para que o sujeito desenvolva satisfatoriamente seu desempenho.

Botomé e Rizzon (1997), assim como Luckesi (2011), destacam que o processo de avaliação de desempenho é constituído por duas etapas e possui como aspecto delimitador o procedimento de intervenção do professor. As etapas são: a) medida; b) procedimento de feedback pelo professor acerca das características do desempenho do estudante. Para Botomé e Rizzon (1997), medir consiste no 
processo de verificar, por meio de testes ou provas, o grau que o estudante está habilitado a executar uma atividade. Essa medida pode ser categórica ou numérica, as quais expressam as características da mensuração. Por outro lado, o processo de avaliar tem como função indicar ao estudante em que ponto está localizado seu desempenho em relação aos objetivos de ensino. Tal localização permite que, em muitas circunstâncias, tanto o estudante como o professor decidam quando, quanto e como alterar o desempenho do estudante em direção ao seu aperfeiçoamento. É importante que o professor forneça feedback informativo e orientador ao estudante. Ele necessita auxiliá-lo a identificar o que está adequado e o que precisa ser aprimorado em seu desempenho e prover condições mais propícias para que esse aprimoramento ocorra. Quando o professor apenas classifica ou rotula o desempenho do estudante, isso pouco o orienta. Cada desempenho necessita receber um feedback imediato, contínuo e específico.

É possível perceber que o processo de avaliar no contexto educacional é constituído pelo processo de medir e por intervenções com o objetivo de aperfeiçoar o desempenho do estudante. Dentre os diferentes autores que apresentam o conceito de avaliação de desempenho, há destaque para o conceito de Luckesi (2011) e Botomé e Rizzon (1997). Os três autores apresentam uma definição de avaliação que parece ser mais promissora, devido à clareza com que apresentam este conceito. Muitas vezes, por ser complexo, o processo de avaliar é confundido com o de medir. Para os autores, o processo de medir faz parte do processo de avaliar (Luckesi, 2011; Botomé \& Rizzon, 1997; McDonald, 2014; Pasquali, 2011). Suas proposições ampliam com clareza as etapas constituintes da avaliação, o que permite tomada de decisões mais acertadas no contexto da educação.

Na literatura da educação, a avaliação pode assumir três funções principais: diagnóstica, formativa e somativa. Essas classificações refletem uma tentativa de caracterizar modalidades de avaliação e que, também, podem ser consideradas funções da medida. Para Haydt (1997), Castillo (2006), Rosa e Maltempi (2006) e García Aretio (2008), quando o professor necessita identificar as características da situação atual do desempenho do estudante e se este apresenta pré-requisitos necessários para desenvolvê-lo, a avaliação diagnóstica ocorre. Ela é considerada importante, pois possibilita ajustes ao longo do programa de ensino. Por outro lado, quando o professor realiza avaliações durante todo o programa para apoiar os estudantes no processo de ensino-aprendizagem em apontamentos de lacunas que precisam ser aprimoradas, avaliações formativas são realizadas. A avaliação que é realizada ao final do curso e com o objetivo de classificar a aprendizagem alcançada pelo estudante, é denominada somativa. Se forem consideradas as proposições realizadas por Luckesi (2011) e Botomé e Rizzon (1997) acerca de avaliação de desempenho, a avaliação somativa pode ser compreendida como sinônimo de medida, pois não são realizadas intervenções após o desempenho do estudante ter sido comparado com o desempenho que era esperado pelo professor. A avaliação diagnóstica também pode ser considerada uma medida do desempenho do estudante se somente for, por meio dela, identificado o estágio inicial do seu desempenho. Já a avaliação formativa pode ser compreendida como avaliação, pois nela a medida é utilizada como um indicativo do desempenho do estudante para que sejam promovidas condições para o seu aprimoramento.

$\mathrm{Na}$ educação a distância (EaD), as avaliações também necessitam ser utilizadas com base em suas funções e de acordo com a especificidade dessa modalidade. Segundo exame feito por Castillo (2006), como o curso em EaD é previamente programado e é mais difícil realizar ajustes durante seu desenvolvimento, a avaliação diagnóstica permite que o professor identifique em quais unidades de aprendizagem o estudante pode iniciar em função do seu repertório inicial no programa. A avaliação formativa é imprescindível na modalidade a distância, pois 
ao avaliar em diferentes momentos o desempenho do estudante, o professor tem acesso contínuo ao seu desenvolvimento. Por fim, a avaliação somativa é utilizada do mesmo modo na educação presencial e seu resultado na educação a distância pode ser pareado com o da avaliação diagnóstica.

Seja na educação a distância ou presencial, o processo de avaliação de desempenho do estudante é uma estratégia recorrente. A diversidade de definições que exprimem esse processo é característica da literatura científica da área. Dentre as definições na literatura, existem algumas cuja ênfase ocorre na interpretação da expressão numérica do desempenho do estudante e outras cujo foco recai sobre as intervenções que são realizadas na direção do aperfeiçoamento do seu desempenho. Qualquer que seja a função de uma avaliação de desempenho, o aspecto comum e de relevância é o de prover informações acerca de processos envolvidos no ensino e no desempenho de alguém. A avaliação com função formativa é de especial interesse, pois consiste em identificar durante todo o programa quais aspectos do desempenho do estudante ainda precisam ser aprimorados. O uso de feedback orientador pelo professor aos estudantes acerca de aspectos de seu desempenho permite com que o estudante o aperfeiçoe. Mas o que caracteriza o feedback orientador? Quais são seus aspectos constituintes?

\section{Feedback do desempenho do estudante como parte constituinte da avaliação de desempenho na educação a distância}

Uma simples expressão da medida é insuficiente para caracterizar o desempenho do estudante. A atribuição de uma nota 2,8 ou 10 ao seu desempenho tem como resultado, muitas vezes e somente, o rótulo. Não basta o professor informar que o estudante obteve nota 5, 6 ou 9. Ou, ainda, dizer que está muito bom seu trabalho. Isso não é suficiente para o estudante identificar qual aspecto de seu desempenho ainda está aquém do esperado, o que é necessário que seja aperfeiçoado e o que é relevante ser mantido. Ao avaliar o desempenho do estudante, o professor necessita fornecer feedback, o mais preciso e inequivocamente possível, a respeito de quais aspectos de seu desempenho necessitam ser aprimorados, ou estão apropriados.

O conceito de feedback começou a ser utilizado na década de 1940 por Norbert Wiener para descrever processos eletrônicos em contexto militar e desde então tem sido aplicado em diversos campos de atuação profissional (Ende, 1983; Wiliam, 2011). O feedback era compreendido como uma informação gerada em um sistema particular com uma finalidade específica, dividindo-se em: positivo e negativo. $\mathrm{O}$ feedback positivo, orientado para uma direção já conhecida e congruente ao sistema. $\mathrm{O}$ feedback negativo, por sua vez, direcionado à tendência oposta ao sistema, com a função de conduzi-lo ao estado anterior a sua modificação (Wiliam, 2011).

Na língua portuguesa, feedback de um processo ou sistema é designado como uma reação a um estímulo que, por sua vez, provoca um novo estímulo, o qual permite a realimentação do processo ou sistema (Dicionário da Língua Portuguesa, 2014). Schermerhorn, Hunt e Osborn (1998) definem feedback como um processo no qual o receptor de uma mensagem comunica outra mensagem ao transmissor. Complementa-se a essas definições, o conceito de feedback encontrado na Teoria Geral do Sistema, em que um sistema tem uma entrada (input) e uma saída (output). Tudo aquilo que o sistema recebe, constitui-se como entrada, enquanto os resultados finais do processamento são as saídas. Nesse processo podem acontecer diversas interações entre os elementos que fazem parte de um sistema, de tal forma que os elementos da saída podem vir a complementar e se tornar elementos da entrada. A esse processo é dado o nome de retroalimentação ou feedback (Lopes, Silva, \& Goulart, 2015).

No contexto do ensino, o feedback, quando é oferecido ao estudante, não apenas possibilita o aperfeiçoamento de seu desempenho apresentado em sala de aula, mas traz decorrências para o 
contexto profissional no qual o estudante atuará. Conforme Boud e Molloy (2013), quando estudante recebe um feedback do professor ou de colegas de estudo, depois de aprimorar seu desempenho em função do feedback recebido, ele tende a ficar sensível às decorrências de seu comportamento no ambiente. Em função disso, ao se tornar um profissional, o estudante passa a monitorar seu desempenho e aprimorá-lo de modo autônomo sem depender necessariamente de um feedback de outra pessoa, o que torna sua atuação mais eficiente e eficaz.

O oferecimento do feedback no contexto da educação é comumente associado à figura do professor quando avalia o desempenho do estudante. Dentre diversos tipos de feedbacks que o professor pode oferecer, Shute (2007) propõe o feedback formativo, entendido como a comunicação de informações ao estudante pelo professor com o objetivo de modificar seu comportamento, cujo resultado seja a melhoria de seu desempenho. O produto do processo de feedback é a melhora no processo comunicativo (Schermerhorn et al., 1998), além de promover desenvolvimento do desempenho do sujeito (Moscovici, 1985). Por meio do feedback, é possível também que os erros sejam corrigidos e que o estudante possa ser motivado a refletir acerca do processo de ensino-aprendizagem e a continuar estudando (Gibbs \& Simpson, 2004).

Quando o professor apresenta feedback ao estudante, há necessidade que ele indique o que ele conseguiu aprender, suas dificuldades e seus equívocos (Ferreira, 2013). Abreu-e-Lima e Alves (2011) afirmam que quando os estudantes não recebem feedback a respeito de seu desempenho muito provavelmente passam a não saber qual aspecto dele necessita aprimorar ou, ainda, não têm conhecimento de quais desempenhos já apresentam de modo adequado, para que possam manter ou repeti-lo. Portanto, o feedback é utilizado como um meio formativo que possibilita a identificação pelo estudante de quais características de seu desempenho necessitam ser melhoradas e/ou mantidas.
Os dados apresentados atestam a necessidade de compreensão dos aspectos que constituem o processo de apresentação de feedback do desempenho de estudantes de programas de educação.

Para que haja melhoria no desempenho do estudante, variáveis como o momento em que o feedback é oferecido e o tipo de informação utilizada necessitam ocorrer sob determinadas condições (Abreu-e-Lima \& Alves, 2011). O momento em que o feedback é apresentado ao estudante constitui um ponto crítico para o desenvolvimento do seu desempenho (Price, Handley, Millar, \& O’Donovan, 2010). $O$ feedback pode ser oferecido imediatamente após a apresentação do desempenho do estudante ou com atraso em relação a ele. $\mathrm{O}$ feedback imediato pode ser definido como aquele oferecido logo após o estudante ter apresentado algum resultado. Nas avaliações somativas, o feedback imediato é oferecido assim que o estudante responde a um teste, por exemplo. O feedback atrasado é definido em relação ao feedback imediato. Ele pode ocorrer minutos, horas ou semanas após o estudante apresentar seu desempenho em alguma tarefa (Shute, 2007).

Existem estudos que demonstram os efeitos do feedback imediato e do feedback atrasado (Kulik \& Kulik, 1988; Butler, Karpicke, \& Roediger III, 2007). Não há consenso na literatura científica acerca de qual é o momento ideal para que sejam oferecidos feedback ao desempenho do estudante. No entanto, Hickey (2013) ao considerar a opinião de estudantes acerca de qual é o momento ideal para receber feedback a respeito de seu desempenho, estes preferiram obter retorno imediato de seu desempenho. Seus comentários foram: “(...) você não tem que esperar para ver suas respostas (...)", "você aprende por seus erros imediatamente" (Hickey, 2013, p.73). Esta preferência também foi encontrada por Bowman e Laurent (2011) ao realizar pesquisas com estudantes, e por Foong, Hassan, Lee e Vadivelu (2015), quando fizeram pesquisas com professores. Portanto, a apresentação do feedback imediato do desempenho do 
estudante é importante no contexto da educação, pois o estudante passa a ficar sob controle do comportamento que necessita aprender e/ou aprimorar logo após ter apresentado o primeiro desempenho.

$\mathrm{O}$ feedback pode ser classificado de diferentes formas em função do tipo de informação contida nele. Quando no feedback há apenas a informação do que o estudante errou e necessita corrigir, este é denominado diretivo. Por outro lado, quando o feedback é constituído por comentários e sugestões que orientem a identificação pelo estudante do que precisa ser revisto para obter melhorias, é nomeado de feedback facilitador (Archer, 2010). Para o feedback ser efetivo é necessário que o estudante o compreenda e esteja disposto e apto para agir com base nas informações que foram indicadas (Price et al., 2010). Santos (2003) sugere que o feedback para ser efetivo necessita ser claro para que o estudante o compreenda; indicar pistas para ações futuras, de modo que o estudante consiga prosseguir com base nele; incentivar o estudante a reanalisar sua resposta; e identificar o que está apropriado. Fook e Sidhu (2014) realizaram um estudo com 192 estudantes de graduação, pós-graduados e professores de uma universidade dos Estados Unidos. Os estudantes afirmaram que um feedback de qualidade necessita indicar, dentre outros aspectos, o que é necessário aprimorar na próxima tarefa e permitir melhoria do aprendizado.

O feedback claro, preciso, completo e exato tende a melhorar o desempenho do estudante, independente da modalidade de educação na qual é utilizado e para qual público se destina. Em um estudo realizado por Butler (1987) foram estudados os efeitos do feedback sobre o desempenho de estudantes na realização de uma tarefa, em função de quatro tipos de informações contidos nele: comentário, classificação, elogio e nenhuma informação. A amostra contou com 200 crianças do $5^{\circ}$ e $6^{\circ}$ anos de escolaridade e apenas o grupo de estudante que recebeu comentários na sua primeira tarefa apresentou uma melhoria estatisticamente significativa no pós-teste, o que indica que feedbacks com comentários tendem a contribuir para o desenvolvimento do desempenho do estudante. Doan (2013), por sua vez, demonstrou em um estudo com 206 estudantes que a utilidade do feedback oferecido pelo tutor, no contexto da educação a distância, dependia de sua qualidade. Quando o feedback era claro, imediato, relevante e construtivo, tendia a melhorar o desempenho do estudante. Por fim, Gusso (2013), realizou uma pesquisa com 66 estudantes em contexto de educação presencial de nível superior. $\mathrm{O}$ autor demonstrou que o uso de feedback informativo escrito e a elaboração de gráficos individualizados de desempenho com frequência semanal parecem ter sido eficientes para a maior parte dos estudantes, o que pode ser evidenciado no aumento da frequência de desempenhos esperados após emissão de feedback informativo pelo professor.

O uso de feedback do desempenho dos estudantes é uma característica presente no processo de avaliação na educação a distância. Nesse contexto, o profissional que interage, presencialmente ou a distância com o estudante e, portanto, oferece feedback a ele, é denominado de tutor (Freitas \& Tarcia, 2014). Ele é o responsável pela mediação entre o que o estudante necessita aprender por meio do material programado e, por esta razão, tem mais acesso aos problemas e suas dificuldades (Nascimento, Santos, \& Araújo, 2012). Abreu-e-Lima e Alves (2011), em um estudo sobre a percepção de tutores sobre a importância do feedback na educação a distância, identificaram uma carga horária de 12 a 14 horas semanais de trabalho dedicadas ao uso de feedback, das 20 horas disponíveis, o que pode ser tomado como indicador de relevância do feedback na educação a distância.

Diferente da educação presencial, na qual ocorre interação direta entre o professor e o estudante, na educação a distância essa interação é mediada por tecnologias, o que traz decorrências no processo de apresentação de feedback pelo tutor que interage a distância com o estudante. Na educação presencial, o professor pode fornecer feedback aos estudantes 
imediatamente à apresentação de seu desempenho, pois este possui como fonte de informações os gestos e a oralidade presentes na própria interação. Por outro lado, como na educação a distância essa interação direta entre o tutor e o estudante não ocorre e tais fontes de informações não são tão frequentes (Castillo, 2006; Garcia Aretio, 2008), os estudantes não têm acesso a dicas gestuais do tutor e dependem apenas de seu feedback textual (Vrasidas \& McIsaac, 1999). Megginson, Mosley e Pietri (1998), ao descreverem as características do feedback oral e escrito, indicam que este permite que o receptor tenha maior atenção à redação da mensagem e que pode interpretá-la no seu ritmo, diferente do feedback verbal em que a mensagem é transmitida em maior velocidade.

Por conta de os estudantes não terem acesso a dicas gestuais do tutor, este necessita estar continuamente atento a sua escrita, tendo em vista que ela consiste como o meio de comunicação com o estudante e que possibilita o feedback. Coutinho, Rodrigues e Ferreira (2012) destacam que entender a qualidade da escrita do feedback no contexto da educação a distância é importante, assim como estar atento às expressões e aos sentidos implícitos e explícitos presentes na comunicação, tendo em vista que elementos envolvidos, desde as pontuações e as formatações do texto, podem atribuir outros significados além daqueles evidentes na escrita. Cabral e Cavalcante (2010) destacam que o tutor necessita evitar frases longas e o uso de ordem inversa da escrita, evitar o uso de linguagem rebuscada ou com formas rígidas e escrever períodos na ordem direta, curtos, completos e com clareza.

Vrasidas e McIsaac (1999) afirmam que o feedback é um importante recurso que parece influenciar a interação entre tutor e estudante. Os autores realizaram uma pesquisa com um tutor e oito estudantes de curso de EaD com a finalidade de investigar características da interação entre eles nesse curso e identificaram que: a) a falta de feedback imediato pode prejudicar e gerar desmotivações pelos estudantes para participarem de discussões online; e b) quando os alunos não recebiam feedback do professor, deixavam de postar mensagens no ambiente virtual. Karal, Çebi e Pekșem (2010) realizaram um estudo acerca do processo de avaliação na educação a distância com 91 estudantes de um programa. Dentre as muitas dificuldades vivenciadas pelos estudantes no processo de avaliação nessa modalidade, em torno de $31 \%$ dos participantes afirmaram que o feedback oferecido a eles era insuficiente, situação esta considerada um problema no curso. Assim, na educação a distância, a apresentação de feedback e de feedback imediato ao desempenho dos estudantes em processos de avaliação é considerado aspecto relevante para os estudantes ao orientá-los no desenvolvimento de seu desempenho.

Os responsáveis pela avaliação na educação a distância necessitam considerar que a apresentação de feedback aos estudantes acerca de seu desempenho, independente das funções específicas para quais a avaliação está sendo feita, oportunizará ao estudante aperfeiçoá-lo. Pré e pós-testes são dois procedimentos de avaliação utilizados conjuntamente na educação a distância cujo objetivo é identificar o desempenho inicial e final do estudante no curso (Delucchi, 2014). O pré e pós-teste constituem avaliação denominada diagnóstica e somativa, respectivamente (Ribeiro \& Lopes, 2006). Ribeiro e Lopes (2006) realizaram um estudo que teve, como um dos objetivos, avaliar a opinião dos estudantes sobre o curso de educação continuada de tratamentos de feridas destinados a enfermeiros na modalidade a distância, e realizar e analisar as avaliações diagnósticas (pré-teste), processuais e somativa (pós-teste). Os autores mostraram que 16 de 17 estudantes obtiveram melhora na sua nota se comparada à do pré-teste. Todos os participantes consideraram que durante o curso, por meio das avaliações, puderam ter conhecimento acerca de quais aspectos do seu desempenho ainda precisavam ser aprimorados 
e 16 estudantes consideraram que por meio das avaliações puderam identificar o quanto seu desempenho foi desenvolvido.

Quanto à percepção dos estudantes acerca da decorrência do processo de avaliação formativa em relação ao seu desempenho, Quintana e Fernandes (2014) realizaram um estudo com estudantes da educação técnica a distância de nível médio (educação profissional) do curso de Administração acerca dos exercícios de avaliação contidos no Ambiente Virtual de Aprendizagem (AVA). Para os participantes, a realização das tarefas no AVAé de extrema importância para a fixação do assunto estudado ao longo da disciplina; outro aspecto relevante indicado pelos estudantes é acerca do papel da tarefa em estimulá-los ao estudo diário e não apenas para obtenção de notas. Tais resultados são corroborados pelo estudo de Quintana e Quintana (2012), com 52 estudantes, o qual os autores afirmaram que as tarefas virtuais representaram elemento indispensável na educação a distância, pois permitiram repercussões significativas no processo de ensino-aprendizagem na compreensão do assunto disponibilizado e na possibilidade de que erros cometidos pudessem ser corrigidos. Os resultados das pesquisas indicaram que uma das consequências da avaliação de desempenho é que o estudante tem acesso, quando este é viabilizado, às características de seu desempenho que ainda necessitam de aperfeiçoamento, independente com qual função a avaliação é utilizada.

O uso de feedback é um aspecto constituinte do processo de avaliar presente na educação a distância. Mais do que o professor/tutor selecionar um tipo de avaliação em relação às funções que esta pode assumir, ele necessita perceber que, ao avaliar o desempenho do estudante, precisa garantir feedback orientador a ele. Afinal, se é necessário que os estudantes aperfeiçoem continuamente seu desempenho, este necessita receber um sinalizador do ambiente onde está inserido a respeito do quão adequado está seu desempenho, o que necessita ser mantido e o que ainda é necessário aperfeiçoar.
Caso contrário, estudantes ficarão igual a Maria da epígrafe desse artigo, sem saber o que fazer para melhorar sua capacidade de modificar o ambiente onde vivem e produzir consequências gratificantes para a comunidade em geral.

\section{Considerações finais}

O objetivo deste artigo foi demonstrar os aspectos constituintes do processo de avaliação de desempenho do estudante e sua relação com o uso feedback na modalidade da educação a distância. Foram examinadas definições teóricas e dados empíricos por meio de uma revisão da literatura. Aspectos teóricos e conceituais sobre medir e avaliar foram discutidos inicialmente para embasar a importância do uso de feedbacks de desempenho de estudantes no processo de avaliação de desempenho.

Avaliar é um processo constituído por duas etapas: 1 . medir, que implica na atribuição de números ou palavras ao desempenho do estudante para 2. intervir na direção de seu aprimoramento. Há três tipos de avaliações existentes na literatura, diagnóstica, formativa e somativa. Apenas na avaliação formativa ocorre, considerando sua definição, a segunda etapa do processo de avaliar, o que traz implicações para o papel do professor (que muitas vezes, sem ter clareza do que seja avaliação, apenasmede o desempenhodo estudante no lugar de avaliá-lo).

Um dos procedimentos de intervenção utilizados no âmbito da avaliação é o uso de feedback. Independente do público que se destina e da modalidade de educação, esse necessita ser claro, preciso, completo, exato e apresentado de modo mais imediato possível, para que o estudante tenha clareza acerca de quais aspectos de seu desempenho necessita aprimorar, manter ou ainda desenvolver.

No contexto da educação a distância, o tutor, enquanto profissional responsável pela mediação entre o curso e o estudante, é quem acompanha e oferece feedbacks ao desempenho do estudante no 
contexto de avaliação. Por isso, é importante que esse profissional seja capacitado para interagir por meio da escrita com os estudantes: seu texto necessita ser claro, direto, evitar expressões implícitas e utilizar pontuações de forma apropriada.

Como sugestões para futuros estudos, é importante que, ao se discutir processos de avaliação independente do contexto no qual ocorre, seja explicitada a perspectiva teórica que o pesquisador assumirá, uma vez que a diversidade de definições acerca do que seja avaliação e os aspectos que a constituem é característica da literatura da área. Sugere-se também que seja produzido conhecimento não apenas acerca do feedback do desempenho do estudante, mas também sobre feedback por parte dos estudantes e tutores aos profissionais responsáveis pelos cursos de educação a distância, como produto do processo de avaliar.

Diante do exposto, para que outras relações possam ser estabelecidas, para que outras relações possam ser estabelecidas, sugere-se a realização de uma revisão sistemática com esse tema. Com uma revisão sistemática, os resultados podem ser organizados de forma cronológica, e a quantidade de artigos por tema e contexto pode ser identificada, o que pode fornecer um conhecimento ainda mais completo sobre o assunto.

\section{Referências}

Abreu-e-Lima, D. M. \& Alves, M. N. (2011). O feedback e sua importância no processo de tutoria a distância. Pro-Posições, 22(2), 189-205.

Alexim, J. C. \& Lopes, C. L. (2003). A certificação profissional revisitada. Boletim Técnico do Senac, Rio de Janeiro, 29(3), 3-15, set./dez.

Archer, J. C. (2010). State of the science in health professional education: effective feedback. $\mathrm{Me}$ dical Education, 44, 101-108.

Botomé, S. P. (1981). Objetivos comportamentais no ensino: A contribuição da análise experimental do comportamento. (Tese de Doutorado, Universidade de São Paulo, São Paulo).
Botomé, S. P. \& Rizzon, L. A. (1997). Medida de desempenho ou avaliação da aprendizagem em um processo de ensino: práticas usuais e possibilidades de renovação. Chronos, 30(1), 7-34.

Botomé, S. P. \& Kubo, O. M. (2002). Responsabilidade social dos programas de pós-graduação e formação de novos cientistas e professores de nível superior. Interação em Psicologia, 6(1), $81-110$

Boud, D. \& Molloy, E. (2013). What is the problem with feedback? In D. Boud \& E. Mollow (eds.). Feedback in higher and professional education:Understanding it and doing it well (pp. 1-10). New York: Routledge.

Bowman, T. G. \& Laurent, T. (2011). Immediate feedback and learning in athletic training education. Athletic Training Education Journal, 6(4), 202-207.

Butler, A, C., Karpicke, J. D., \& Roediger III, H. L. (2007). The effect of type and timing of feedback on learning from multiple-choice tests. Journal of Experimental Psychology: Applied, 13(4), 273-281.

Butler, R. (1987). Task-involving and ego-involving properties of evaluation: Effects of different feedback conditions on motivational perceptions, interest and performance. Journal of Educational Psychology, 79(4), 474-482.

Cabral, A. L. T. \& Cavalcante, A. F. (2010). Linguagem escrita. In A. Carlini \& R. M. Tarcia (Coords.), 20\% a distância: e agora? Orientações práticas para o uso da tecnologia de educação a distância (pp. 53-80). São Paulo: Pearson Education do Brasil.

Caixeta, L. V. \& Silva, I. I. C. (2014) Avaliação psicológica: possibilidades e desafios atuais. Revista Perquirere, 11(2), 218-237.

Castillo, Q. (2006) Evaluación del aprendizaje em la educación a distancia “en línea”. RED. Revista de Educación a Distancia, (6), 1-15.

Chacón, F. (1994). Asesoría magistral encriterios e indicadores de calidad de la educación superior abierta y a distancia. Primera Reunión Latinoa- 
mericana a Distancia de Educación Superior Abierta y a Distancia. Noviembre de 1994. Retrieve from http://herzog.economia.unam. $\mathrm{mx} /$ enlinea/sualin/Evead/Lecturas/Lectura4.doc Coutinho, A. M. A. M., Rodrigues, L. M. S., \& Ferreira, S. M. B. (2012). Uma ferramenta discursiva: os feedbacks nos fóruns de discussão. In Software livre e Anais do IX Encontro Virtual de Documentação e VI Congresso Internacional de Linguagem e Tecnologia Online.

Cruz, R. M., Alchieri, J. C., \& Sardá, J. J. (2002). Avaliação e Medidas Psicológicas: produção do conhecimento e da intervenção profissional. São Paulo: Casa do Psicólogo.

De la Orden, A. (2000). La función optimizante de la evaluación de programas evaluativos. Revista de Investigación Educativa, 18(2), 381-389.

Delucchi, M. (2014). Measuring student learning in social statistics: A pretest- posttest study of knowledge gain. American Sociological Association, 42(3), 1-9.

Dicionário da Língua Portuguesa. (2014). Dicionário da Língua Portuguesa. Porto: Editora Porto.

Doan, L. (2013). Is feedback a waste of time? The students' perspective. Journal of Perspectives in Applied Academic Practice, 1(2), 3-10.

Ende, J. (1983). Feedback in clinical medical education. Medical Education, 250(6), 777-781.

Esteves, O. P. (1973). Testes, medidas e avaliação. Rio de Janeiro: Arte \& Indústria.

Ferreira, C. A. (2013). Contributos para uma prática avaliativa reguladora da aprendizagem. $E D U$ SER: revista de educação, 5(2), 18-34.

Foong, C. C., Hassan, H., Lee, S. S., \& Vadivelu, J. (2015). Using students' formative feedback to advocate reflective teaching. Medical Education, 49, 531-541.

Fook, C. Y. \& Sidhu, G. K. (2014). Assessment practices in higher education in United States. Procedia - Social and Behavioral Sciences, 123, 299-306.
Freitas, A. D. \& Tarcia, R. M. L. (2014). Avaliação de indicadores para a prática de tutoria. In Anais do $20^{\circ}$ Congresso Internacional ADEB Educação a Distância, 1-10.

García Aretio, L. (2008). Evaluación em formatos no presenciales. Madrid: Editorial Del BENED.

Gibbs, G. \& Simpson, C. (2004). Conditions Under Which Assessment Supports Students' Learning. Learning and Teaching in Higher Education, 1, 3-31.

Gusso, H. L. (2013). Avaliação da eficiência de um procedimento de apresentação semanal de consequências informativas ao desempenho de aluno em nivel superior. (Tese de Doutorado, Universidade Federal de Santa Catarina, Florianópolis).

Haydt, R. C. C. (1997). Avaliação do processo ensino-aprendizagem. São Paulo: Ática.

Hickey, D. (2013).Case study: a comparison of immediate and delayed feedback, in the context of online testing with fourth class students. (Master of Arts, University of Limerick, Ireland). Retrieve from http://ulir.ul.ie/handle/10344/3579.

Karal, H., Çebi, A., \& Pekșem, M. (2010). Student opinions about the period of measurement and evaluation in distance education: the difficulties. Procedia Social and Behavioral Sciences, 9, 1597-1601.

Kulik, J. A. \& Kulik, C. C. (1988). Timing of feedback and verbal learning. Review of Educational Research, 58(1), 79-97.

Lamonato, C. (2011). Comportamentos profissionais de empregados em período de experiência objeto de avaliação de desempenho em uma agroindústria. (Dissertação de Mestrado, Universidade Federal de Santa Catarina, Florianópolis).

Lopes, L. G. N., Silva, A. G., \& Gourlart, A. C. O. (2015). A teoria geral do sistema e suas aplicações nas ciências naturais. Natureza online, 13(1), 1-5. 
Luckesi, C. C. (2011). Avaliação da aprendizagem: componente do ato pedagógico. São Paulo: Cortez.

Mager, R. F. (1981). Medindo os objetivos de ensino: ou "conseguiu um par adequado?". Rio de Janeiro: Globo.

McDonald, R. (2014). Test theory a unified treatment. New York: Routledge.

Megginson, L. C., Mosley, D.C., \& Pietri, P. H. Jr. (1998). Administração. São Paulo: Harbra.

Moscovici, F. (1985). Desenvolvimento interpessoal. Rio de Janeiro: LTC - Livros Técnicos e Científicos Editora S.A.

Nascimento, D. S., Santos, M. B., \& Araújo, M. S. (2012). Atuação do professor-tutor no contexto da democracia cibercultural na educação a distância. SIED - Simpósio Internacional de Educação a Distância e EnPED - Encontro de Pesquisadores em Educação a Distância.

Pasquali, L. (2011). Psicometria: teoria dos testes na psicologia e na educação (5 $5^{\text {a }} \mathrm{ed}$.). Petropolis: Vozes.

Price, M., Handley, K., Millar, J., \& O’Donovan, B. (2010). Assessment \& Evaluation in Higher Education, 35(3), 277-289.

Quintana, A. C. \& Quintana, C. G. (2012). A execução das tarefas virtuais no contexto da educação a distância: um estudo em um curso de graduação em administração. Revista Gestão Universitária na América Latina - GUAL, 5(2), 277-297.

Quintana, A. C. \& Fernandes, V. L. P. (2014). Percepção do estudante de educação técnica a distância de nível médio sobre a realização das tarefas avaliadas no ambiente virtual. RIED, 17(2), 127-150.

Ribeiro, D. (1969). A universidade necessária. Rio de Janeiro: Paz e Terra.

Ribeiro, M. A. S \& Lopes, M. H. B. M. (2006). Desenvolvimento, aplicação e avaliação de um curso à distância sobre tratamento de feridas. Revista Latino-America de Enfermagem, 14(1),77-84.

Rosa, M. \& Maltempi, M. V. (2006). A avaliação vista sob o aspecto da educação a distância. Ensaio: avaliação e políticas públicas em educação, 14(50), 57-76.

Santa'anna, I. M. (2002). Por que avaliar? Como avaliar? Critérios e instrumentos. Rio de Janeiro: Vozes.

Santos, L. (2003). Avaliar competências: uma tarefa impossível? Educação e Matemática, (74), 1621. Retrieved from http://www.educ.fc.ul.pt/ docentes/msantos/comp.pdf

Schermerhorn, J., Hunt, J. G., \& Osborn, R. N. (1998). Comunicação. In J. Schermerhorn, J. G. Hunt, \& R. N. Osborn. Fundamentos de comportamento organizacional (pp. 239-252). Porto Alegre: Bookman.

Shute, V. J. (2007). Focus on Formative Feedback. ETS Research Reports.

Vrasidas, C. \& McIssac, M. S. (1999). Factors influencing interaction in an online course. American Journal of Distance Education, 8(2), 6-29.

Wiliam, D. (2011). What is assessment for learning? Studies in Educational Evaluation, 37, 3-14.
Recebido: junio 17, 2015 Aprovado: septiembre 25, 2015 
